\title{
eNOS and Diabetic Nephropathy
}

\section{Feng Li' and Nobuyuki Takahashi ${ }^{1,2,3 *}$}

${ }^{1}$ Dept of Pathology and Laboratory Medicine, The University of North Carolina, Chapel Hill, NC 27599, USA

${ }^{2}$ Dept of Cell and Molecular Physiology, The University of North Carolina, Chapel Hill, NC 27599, USA

${ }^{3}$ Graduate Schools of Pharmaceutical Sciences and Medicine, Tohoku University, Sendai 980-8578, Japan

\begin{abstract}
Diabetic nephropathy (DN) is the leading cause of end stage renal disease (ESRD) and the number of patients with DN has been increasing rapidly. Development of new effective therapeutic strategies of DN is slow at least partly because of a lack of animal models that recapitulate the features of human DN. Human variants of the endothelial nitric oxide synthase gene (eNOS, NOS3) that produce reduced amounts of nitric oxide (NO) are positively associated with DN, although proof of causation is lacking. Recently, several investigators have established animal models of advanced DN using mice unable to synthesize eNOS and have demonstrated that eNOS -/- mice with diabetes develop severe nephropathy. It has been also shown that a high fat diet worsens the DN of the diabetic mice lacking eNOS. However, complete absence of eNOS has not been reported in humans, although reduced levels are not infrequent. Accordingly, heterozygous eNOS +/- mice have been made diabetic and they demonstrated that the decrease in eNOS/ NO comparable to that of NOS3 polymorphisms is sufficient to cause exacerbation of DN. Increased expression of tissue factor (TF), initiator of coagulation, plays a significant role in the DN of the eNOS -/- diabetic mice, as well as in diabetic mice having wild type eNOS. Strategies to ameliorate hypercoagulability could be useful for treatment of DN.
\end{abstract}

Keywords: Mouse; Tissue factor; Inflammation

\section{Introduction}

More than $30 \%$ of all diabetic patients develop diabetic nephropathy (DN) [1], which is the most frequent cause of chronic kidney disease (CKD) and is a risk factor for stroke and heart attack [2]. The number of diabetic patients and those affected by $\mathrm{DN}$ is rapidly increasing [3]. Not all diabetic patients develop DN and the development and severity of DN vary greatly from one patient to another with familial clustering, suggesting that genetic factors play an important role [4]. One of the most well-known changes in genes that exacerbate DN is increased expression of angiotensin converting enzyme (ACE) [5]. Recently, genome wide association studies (GWAS) have identified several susceptibility loci associated with DN [6]. Although all these genetic and epigenetic studies suggest the association between the genes and DN, they do not prove the causality. Different from human studies, animal studies are very informative because their genetic background and environmental factors such as diet can be controlled. It is becoming more and more important to establish animal models, which recapitulate human $\mathrm{DN}$, where causation can be explored.

Increased expression of ACE exacerbates DN in mice [7], indicating that increased ACE expression as in ACE DD genotype causes an exacerbation of DN. Although ACE inhibitors are very effective antihypertensive drugs, mice with mildly increased ACE comparable to individuals with the ACE DD genotype do not have increased blood pressure (BP) or plasma angiotensin II (Ang II) levels [8], indicating that the exacerbation of DN caused by a mild increase in ACE is not due to increased Ang II (Figure 1A).

The resolution of this contradiction is illustrated by the computer simulation shown in Figure 1. The simulation showed that mild inhibition of ACE, as in the Ace one-copy mice (equivalent to $50 \% \mathrm{ACE}$ inhibition), causes an increase in Ang I, which offsets the decrease in ACE so that plasma Ang II and BP stay normal. However, if ACE is extensively inhibited, plasma Ang I levels plateau and further decreases in ACE causes a decrease in Ang II and BP. Mild changes in the expression of ACE, such as those seen in the human polymorphism and in the mouse gene titration experiments, are not sufficient to move the system away from the offset region to where ACE differences affect Ang II levels and BP. ACE inhibitors (ACEI) on the other hand move the system to the region where Ang II levels and BP are decreased. In addition to converting Ang I to Ang II, ACE inactivates bradykinin (BK) and mild increase in ACE comparable to ACE DD genotype decreases $\mathrm{BK}$ by $20 \%$ in mice, suggesting that advanced $\mathrm{DN}$ by mild increase in ACE is likely due to a decrease in BK levels (Figure 1A) [8]. This hypothesis has been confirmed in experiments that diabetic mice lacking type 2 bradykinin receptor (B2R) exhibit accelerated DN compared to wild type diabetic mice [9].

Animal models of Diabetic Complications Consortium (AMDCC), currently Diabetic Complications Consortium (DCC, http://www. diacomp.org/) was set up to develop good animal models of human $\mathrm{DN}$, to investigate in mice the strain differences in $\mathrm{DN}$ and to investigate genes involved in DN [10,11]. The roles of bradykinin receptors were further investigated as part of AMDCC. Mice lacking both B1 and B2 receptors show severe DN $[12,13]$. Since stimulating bradykinin receptors activates eNOS, it is speculated that a decrease in eNOS activity also accelerates DN. Recently, multiple laboratories including ours have demonstrated that diabetic mice lacking eNOS develop human-like nephropathy [14-16].

\section{NOS3 Polymorphisms and DN}

NOS3 gene locates on chromosome 7q36, having 26 exons and 25 introns and is approximately $23.5 \mathrm{~kb}$ in length. NOS 3 polymorphisms leading to reduced NO production have been widely studied and are

*Corresponding author: Nobuyuki Takahashi, Graduate Schools of Pharmaceutical Sciences and Medicine, Tohoku University, 6-3 Aoba Aramak Aoba-ku Sendai, 980-8578, Japan, Tel: +81-22-795-6807; Fax: +81-22-7956839; E-mail: ntakaha@m.tohoku.ac.jp

Received December 23, 2011; Accepted February 23, 2012; Published February 25, 2012

Citation: Li F, Takahashi N (2012) eNOS and Diabetic Nephropathy. J Nephrol Therapeutic S2. doi:10.4172/2161-0959.S2-004

Copyright: (c) 2012 Li F, et al. This is an open-access article distributed unde the terms of the Creative Commons Attribution License, which permits unrestricted use, distribution, and reproduction in any medium, provided the original author and source are credited. 
A.

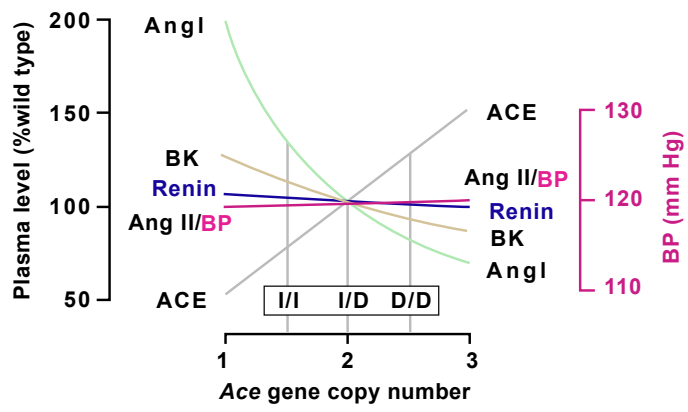

B.
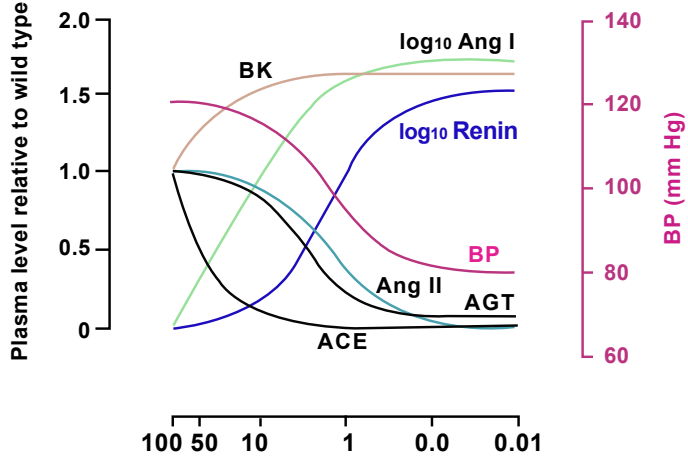

ACE actvity (\%)

Figure 1: Simulations of $A c e$ gene titration and progressive ACE inhibition. (A), ACE gene titration. Mild quantitative changes in the expression of ACE do not affect plasma Ang II level or BP because of the concomitant changes in the level of Ang I. However, plasma bradykinin (BK) level changes with changes in ACE expression. The levels expected in association with the human I/D polymorphism are shown. (B). Effects of ACE inhibition. When inhibition is virtually complete, the simulation shows that: 1) Ang I levels are high and plateaued; 2) Ang II levels are low; 3) BK levels are increased by the virtual absence of ACE; and 4) BP is substantially lowered. (Adopted from reference 8 with permission).

associated with a variety of diseases including essential hypertension [17], coronary artery disease [18] and venous thromboembolism [19]. To date, three common polymorphisms of NOS3 related to DN have been studied broadly. They are: 1) G894T in exon 7 that leads to the replacement of a glutamic acid by aspartic acid at residue 298 (Glu298Asp); 2) 4b/a with a 27 nucleotide variable number of tandem repeats within intron 4 ( $4 \mathrm{a}$ with four repeats and $4 \mathrm{~b}$ with five repeats); 3) $\mathrm{T}-786 \mathrm{C}$ in the promoter region.

\section{Association of the G894T (Glu298Asp) variant with DN}

The $894 \mathrm{~T}$ NOS3 gene produces about $30 \%$ of the amount of NO produced by the more common $894 \mathrm{G}$ [20]. Recent meta-analysis performed by Zeng et al. [21] show that this allele is associated with different effects in different populations. Total of 15 studies involving 6,725 individuals, about half of them are patients with DN, have been analyzed. In control groups, the frequency of $894 \mathrm{~T}$ is higher in Caucasian than in East Asian. However, in Caucasian, there was no association between G894T (Glu298Asp) and DN. In East Asian, on the other hand, this polymorphism is positively associated with DN [21].

\section{Association of the $4 \mathrm{~b}$ /a variant with $\mathrm{DN}$}

The plasma level of nitrate/nitrite, the metabolites of $\mathrm{NO}$, in individuals with the allele is $20 \%$ lower than that of non-carriers [22]. The relationship between the $4 \mathrm{~b} / \mathrm{a}$ variants and $\mathrm{DN}$ is uncertain. A meta-analysis carried out by Zeng et al. [21] showed that there was no association between the $4 \mathrm{~b} / \mathrm{a}$ variant and $\mathrm{DN}$ in global populations, although the association became significant if individuals with microalbuminuria were excluded from the study [21]. A meta-analysis performed by $\mathrm{He}$ et al. [23] likewise, did not find an association between $4 \mathrm{~b} / \mathrm{a}$ and $\mathrm{DN}$ in Caucasians, but their analysis showed a positive association in the global population and East Asian.

\section{Association of the T-786C variant with DN}

This mutation is associated with decreased promoter activity, eNOS transcription and NO production [24]. This allele of NOS3 has been shown to be associated with $\mathrm{DN}$ in global population. The metaanalysis performed by Zeng et al. [21] including 3,045 individuals (about $48 \%$ of them were diabetic patients with $\mathrm{DN}$ ) showed that an overall association between T-786C and DN. Unlike two other variants, the positive association was also found in Caucasian when the analysis included only ethnic groups originated from Europe.

\section{Features of human DN}

Before discussing DN of mice lacking eNOS, we will briefly summarize the features of human DN.

Glomerular lesions: In the earliest stage of DN, glomerular hyperfiltration and hypertrophy develop in patients $[25,26]$ and there is some association between early onset hyperfiltration, hypertrophy and the subsequent development of other pathological changes of DN [2527]. Thickening of the glomerular basement membrane (GBM) is also one of the first measurable changes in diabetic patient 1.5-2.5 years after the onset of type I diabetes [28,29]. Thickening of GBM results from accumulation of extracellular matrix (ECM) proteins including collagen type IV, type $\mathrm{V}$ and fibronectin [25,30]. The accumulation of ECM also contributes to mesangial expansion [25], although initiation of GBM thickening and mesangial expansion occur independently at variable rates [26]. When mesangial expansion exceeds a Vv (volume of mesangium/volume of glomerulus) greater than $37 \%$, patients will have some manifestation of nephropathy, such as microalbuminuria, decreased glomerular filtration rate (GFR) [31-33]. The progression of the mesangial lesions leads to Kimmelstiel-Wilson nodules [25] and the formation of Kimmelstiel-Wilson nodules is considered to indicate that $\mathrm{DN}$ is progressing to the next stage [25]. Together, these lesions finally result in glomerulosclerosis. The mechanisms underlying these pathological changes of DN are still largely unknown, although Nakagawa and we have respectively proposed "vascular endothelial growth factor (VEGF) -eNOS/NO uncoupling" and "Tissue Factor (TF)/hypercoagulability-inflammation" are involved, as discussed later in this review.

Tubulointerstitial lesions: Like thickening of GBM, thickening of the tubular basement membrane is due to excess ECM resulting from increased production and/or decreased degradation. This pathological change is detectable parallel to GBM thickening [34]. As DN proceeds, interstitial fibrosis and tubular atrophy also occur and glomerular lesions eventually lead to ESRD [35]. Presence of macrophages and T-lymphocytes in the interstitium [36] suggests that inflammation plays a role in tubulointerstitial lesions. 
Vascular lesions: Hyalinosis of both afferent and efferent arterioles occurs within a few years after the onset of diabetes [37]. Stout et al. [38] suggest that hyalinosis of efferent arterioles is relatively specific for $\mathrm{DN}[25]$ and is a lesion which distinguishes $\mathrm{DN}$ from hypertensive nephropathy $[25,39]$.

\section{Mouse Models of DN: the Lessons from Diabetic eNOS -/- Mice}

In general, the mouse is a relatively poor model of $\mathrm{DN}$, only showing partial early stages of the lesions observed in human DN. In 2009, AMDCC proposed a validation criteria of mouse models of DN (http://www.diacomp.org/), including 1) functional changes: $50 \%$ or more decrease in GFR over the lifetime and $\geq 10$ fold increase in urinary albumin excretion compared with controls with the same gender, age and strain. 2) structural changes: basement membrane thickening by electron microscopy; advanced mesangial matrix expansion with or without nodular sclerosis and mesangiolysis; tubulointerstitial fibrosis and any degree of arteriolar hyalinosis [11]. In the past five years, multiple laboratories have independently reported that advanced DN develops in mice lacking eNOS. The different groups have used different regimens to establish DN models in mice lacking eNOS and these models, to some extent, meet the criteria defined by AMDCC. Table 1 summarizes the features of DN of these models.

\section{Low-dose STZ (streptozotocin), high fat (HF) diet regimen}

We have initiated diabetes in male eNOS -/- and their littermate WT mice using low-dose STZ (40 mg/kg, 5 days) based on DCC protocol. Half of the mice were fed a HF diet. The combination of eNOS deficiency and HF diet causes severe pathological changes in the kidneys of diabetic mice, although their plasma glucose levels were not higher than those of WT diabetic mice. Diabetic eNOS -/- mice fed HF diet have increased urinary albumin excretion as early as 12 weeks after the onset of diabetes. At 24 weeks of diabetes, these mice show advanced DN, which meets almost all the criteria defined by DCC. Urinary albumin excretion increased to almost 5 times of WT diabetic mice and creatinine clearance was $50 \%$ of control mice, indicating progressive renal insufficiency. Strikingly, these mice show severe mesangiolysis and glomerulosclerosis (Figure 2A). They also have tubulointerstitial fibrosis, mesangial expansion and thickening of the GBM [14]. Kanetsuna et al. [40] also used the low-dose STZ regimen to investigate the role of eNOS/NO in DN using eNOS -/- mice with the same genetic background as ours [37]. Their mice were fed normal chow and their findings are consistent with ours.
After establishing the mouse model of DN using STZ-diabetic eNOS -/- mice, we investigated the mechanism causing severe DN when eNOS is absent. Besides vasodilatation, eNOS/NO plays an important role against oxidative stress [41], inflammation [42] and coagulation [43]. Since micro-thrombi were found in glomeruli of diabetic eNOS -/mice fed HF diet, it was hypothesized that activated coagulation system may be responsible for aggravating $\mathrm{DN}$ in these mice. Indeed, diabetic eNOS -/- mice fed HF diet had the most abundant fibrin deposits in their glomeruli, although glomeruli from all diabetic mice in this study were positive for fibrin. Kidneys from diabetic eNOS -/- mice have markedly increased TF expression and activity. Immunoreactive TF was almost exclusively expressed in macrophages/monocytes infiltrated in glomeruli (Figure 2B). Increased expression of TF precedes overt DN and administration of an anti-mouse TF neutralizing antibody to diabetic mice reduces the expression of inflammatory and fibrogenic genes, to a greater extent in eNOS -/- mice as shown, for instance, in Figure 2C. There are intricate relationships among eNOS/NO, TF and DN (Figure 3). NO inhibits [44] and HF stimulates [45] the NF$\kappa \mathrm{B}$ pathway, one of the most important regulators of TF expression. Increased TF expression in the kidney activates the coagulation cascade, leading to the production of FVIIa, FXa and thrombin. These proteases can activate protease-activated receptors, which may enhance DN [46]. TF can also mediate inflammation. Interestingly, TF was only detected in monocytes/macrophages in glomerular mesangial area of our mice. Although immunoreactive TF was not detected in mesangial cells in vivo, mesangial cells are suggested to be responsible for mesangial expansion and glomerulosclerosis in DN. When macrophage in the mesangial area express TF and activate the coagulation cascade, FVIIa, FXa and thrombin will be produced, which could increase the expression of inflammatory genes including MCP1 in mesangial cells, possibly through binding to protease activated receptors. Hypercoagulability and inflammation together likely make a vicious circle in exacerbating $\mathrm{DN}$.

\section{eNOS +/- Akita and eNOS -/- Akita mice}

Although lack of eNOS exacerbates DN in mice, complete lack of gene products in general human population does not likely cause multigenic diseases including DN. Rather an accumulation of small changes in multiple genes more likely causes these diseases. Accordingly, even though individuals with NOS3 polymorphisms that are associated with mild reduction of NO production are associated with DN, whether the mild decrease in NO production from eNOS is sufficient to cause $\mathrm{DN}$ is not clear from the studies of homozygous eNOS -/- mice. To tackle this problem we took advantage of hybrid

\begin{tabular}{|c|c|c|c|c|c|c|}
\hline & eNOS-/- $d b / d b$ & eNOS-/- high-dose STZ & eNOS-/- low-dose STZ & $\begin{array}{c}\text { eNOS-/- low-dose } \\
\text { STZ, HF }\end{array}$ & eNOS-/- Akita & eNOS+/- Akita \\
\hline & Ref.16 & Ref.15 & Ref.40 & Ref.14 & Ref.47 & Ref.47 \\
\hline u-albumin & $\uparrow$ & $\uparrow$ & $\uparrow$ & $\uparrow$ & $\uparrow$ & $\uparrow$ \\
\hline GFR & $\downarrow$ & not determined & $\downarrow$ & $\downarrow$ & $\downarrow$ & $\uparrow$ \\
\hline GBM thickening & + & + & + & + & + & + \\
\hline mesangial expansion & + & + & + & + & + & + \\
\hline mesangiolysis & + & + & + & + & + & + \\
\hline nodular sclerosis & + & + & + & + & + & + \\
\hline tubulointerstitial fibrosis & - & + & - & + & - & - \\
\hline arteriolar hyalinosis & + & + & not determined & - & - & - \\
\hline
\end{tabular}

Table 1: Features of diabetic nephropathy in mice with decreased eNOS expression. 
A.

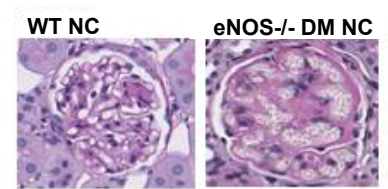

B.

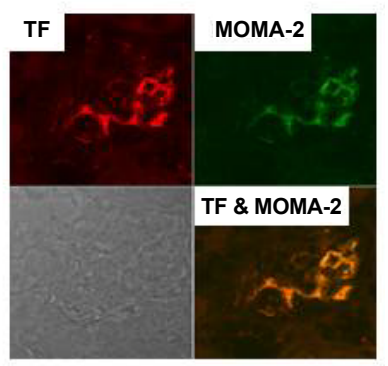

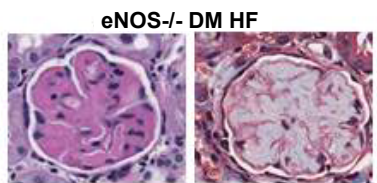

C.

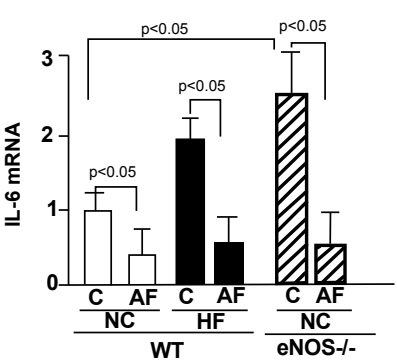

Figure 2: Diabetic nephropathy of eNOS-/- mice after 6 months of diabetes induced by streptozotocin. (A). Glomerular morphology.WT NC: a glomerulus from a wild type non-diabetic mouse fed a normal chow; eNOS-/DM NC shows mesangiolysis; eNOS-/- diabetic mice fed a high fat diet show severe glomerulosclerosis. (B). Immunostaining against TF and monocytes/ macrophages (MOMA-2) in a diabetic eNOS-/-mouse fed a HF diet. The left lower panel is a differential interference contrast image of the glomerulus. (C). Kidney IL-6 mRNA expression in diabetic mice. C: control; AF: anti-mouse TF neutralizing antibody AF3178 administered i.p. once at 5 weeks of STZ-induced diabates, and the animals were sacrificed for analysis 4 days later. (Adopted from reference 11 with permission).

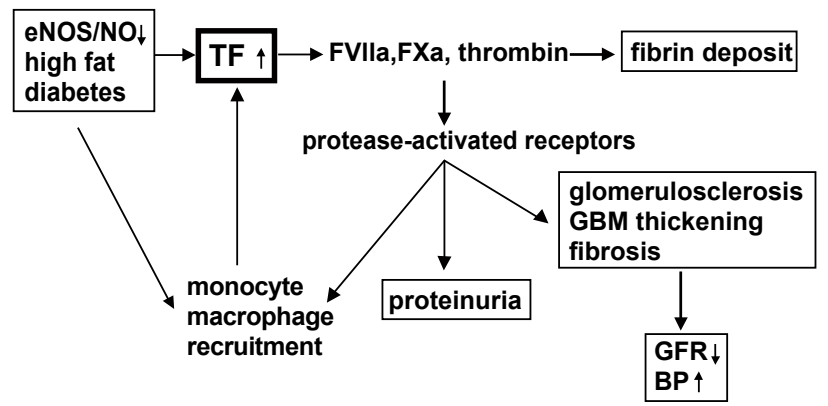

Figure 3: Tissue Factor (TF) contributes to pathogenesis of DN (hypothesis). A decrease in eNOS/NO, high fat diet and diabetes all stimulate TF expression. Increased TF expression in the kidney (observed in monocytes/ macrophages in glomerular mesangial area) activates the coagulation cascade, leading to the production of FVIIa, FXa and thrombin. These proteases can activate protease-activated receptors, which may enhance DN, inflammation and recruit monocyte/macrophages. Hypercoagulability and inflammation together likely make a vicious circle and exacerbate DN.

vigor and genetic uniformity of the $\mathrm{F} 1$ progeny $(\mathrm{eNOS}+/+,+/-$, or -/- with or without Akita diabetic mutation) of a cross between heterozygous 129SvEvTac eNOS +/- inbred females and C57BL/6 eNOS +/- inbred males carrying the dominant Akita diabetogenic mutation in Ins 2 gene. In order to carry out this type of experiment, it is extremely important to obtain mice of the critical genotypes at or close to the same time, so that one can measure parameters in a way that minimizes seasonal changes, dietary differences, any differences in the degrees of backcrossing, or unplanned differences in genotypes. Note that both parents, although inbred, are heterozygotes for the eNOS knockout, which are almost always healthier and have more offspring than homozygous mutants. The offspring are F1 hybrids (genetically as uniform as inbreeds but hardier). Note also that this mating produces the all of possible genotypes as littermates. Studying

all six genotypes is very informative. If heterozygotes are included, the functional consequences of different levels of expression of eNOS on diabetic complications can be determined. If WT and mutant eNOS mice are studied on both diabetic and non-diabetic backgrounds, additive, super-additive or sub-additive interactions between eNOS and diabetes can be detected. More generalized precautions can be found at DCC website: <http://www.diacomp.org/shared/showFile. asp $x$ ? doctypeid $=3 \&$ docid $=79>$.

The expression of eNOS in glomeruli of the heterozygous eNOS $+/$ - mice is approximately $30 \%$ of that of WT, which is comparable to NO production of TT genotype compared to that of GG genotype of NOS3 polymorphism G894T [47]. Using these mice it was shown that mild decrease in eNOS expression in eNOS +/- mice is sufficient to exacerbate DN. As demonstrated in eNOS -/- STZ mice, eNOS +/Akita and eNOS -/- Akita mice develop DN likely due to activation of coagulation system [47].

\section{High -dose STZ regimen}

Nakagawa's group first reported diabetic renal injury using high dose STZ (100mg/kg, 2 days) in eNOS -/- mice [15]. The pathological changes in these mice also meet the criteria of DN proposed by DCC. The survival rate dramatically decreased in diabetic eNOS -/- mice compared to WT diabetic mice 5 months after inducing diabetes. These mice had higher BP than non-diabetic eNOS -/- mice, increased urinary albumin excretion and an elevation of BUN. The early features of DN in these mice include mesangial expansion and thickening of basement membrane. In addition, characteristics of advanced DN also developed after 5 months of diabetes, including mesangiolysis, capillary microaneurysms, nodular lesions and glomerulosclerosis. These authors suggested that uncoupling of VEGF with eNOS/NO can cause DN [48-50], because surprisingly high expression of VEGF was observed in diabetic eNOS -/- mice and because uncoupling causes excessive endothelial cell proliferation in response to VEGF in vitro [48]. They also found that diabetic eNOS -/- mice have macrophage infiltration, which is associated with severe glomerular injury. They suggest uncoupling of VEGF-eNOS/NO plays a role in macrophage migration [51].

\section{eNOS -/- db/db mice}

Zhao et al. [16] have introduced eNOS deficiency into $d b / d b$ mice to study diabetic renal injuries in a model of type II diabetes. Although blood glucose levels of eNOS -/- $d b / d b$ mice did not differ from those of $d b / d b$ mice, lack of eNOS undoubtedly exaggerates DN including dramatic albuminuria, arteriolar hyalinosis, increased GBM thickness, mesangial expansion, mesangiolysis and focal segmental and early nodular glomerulosclerosis. In addition, eNOS -/- $d b / d b$ mice exhibit decreases in GFR to levels $<50 \%$ of that in $d b / d b$ mice.

\section{Therapeutic approaches in eNOS -/- diabetic mice}

Nakagawa's group and Harris's group have tested the effects of several drugs on DN of their eNOS -/- diabetic mice. Nakagawa's group has found that hydralazine, an antihypertensive drug, significantly lowered BP and ameliorated glomerular injuries in their eNOS -/- STZdiabetic mice. But albuminuria and tubulointerstitial injury were not improved by hydralazine [52]. They also reported that spironolactone (a mineralocorticoid receptor antagonist) is superior to enalapril (an ACE inhibitor) or telmisartan (an angiotensin receptor blocker) in protecting eNOS -/- STZ diabetic mice, evidenced by reduction in BP, 
urinary albumin excretion and renal injury [53]. Harris' group has treated eNOS $-/-\mathrm{db} / \mathrm{db}$ mice with captopril (ACEI) and triple drugs (hydralazine, resperine, hydrocholorothiazide) at doses that reduce BP to the similar levels and found that captopril was more beneficial than triple therapy in ameliorating DN including urinary albumin excretion and glomerular injury. These data suggest that ACEIs have additional beneficial effects on eNOS $-/-\mathrm{db} / \mathrm{db}$ mice, which is beyond lowering $\mathrm{BP}$. The authors suggest that there is unknown mechanism(s) underlying the renoprotective benefit of RAS blockade besides increasing eNOS/ NO [54]. Different kinds of diabetic eNOS -/- mouse models reviewed here provide strong evidence that eNOS/NO play a critical role in DN. Following this reasoning, NO donors or reagents that activate or prolong the second message of NO, such as cGMP could be useful for treatment of DN. Indeed, Kuno et al. [55] reported that sildenafil (a PDE5 inhibitor) attenuated DN in Otsuka Long-Evans Tokushima Fatty rats.

\section{Conclusion}

The diabetic eNOS -/- mice are an excellent model of human DN. Although hypercoagulability is well known in diabetes, it has not been widely considered to cause DN. Because hypercoagulability caused by increased TF exacerbates DN, treatment against hypercoagulability might be useful for treating or preventing DN besides RAS blockade.

\section{Acknowledgment}

Authors thank Drs. Oliver Smithies, Nobuyo Maeda, Sadayoshi Ito and Hirosh Sato for their support. This work was supported by grants from American Heart Association (0265464U, 0855335E), the National Institutes of Health (P30DK56350, P30 ES10126) and Grant-In-Aid from The Japan Society of Promotion of Science (JSPS, 22890016), Funds from the Department of Pathology and Laboratory Medicine at The University of North Carolina at Chapel Hill, Funds from Center for Advanced and Integrated Renal Science, Tohoku University Graduate School of Medicine, Funds from Miyagi Association of Kidney Disease, Funds from Banyu Life Science Foundation International, the committee grant from the Japanese Society of Nephrology and a grant-in-aid from the Research Group on Progressive Renal Disease from the Ministry of Health, Labor and Welfare, Japan.

\section{References}

1. Perkins BA, Krolewski AS (2005) Early nephropathy in type 1 diabetes: a new perspective on who will and who will not progress. Curr Diab Rep 5: 455-463.

2. Brown WV (2008) Microvascular complications of diabetes mellitus: renal protection accompanies cardiovascular protection. Am J Cardiol 102:10-13.

3. Williams ME (2010) Diabetic CKD/ESRD 2010: a progress report?. Semin Dial 23: $129-133$

4. Bowden DW (2002) Genetics of diabetes complications. Curr Diab Rep 2: 191 200 .

5. Sayed-Tabatabaei FA, Oostra BA, Isaacs A, van Duijn CM, Witteman JC, et al. (2006) ACE polymorphisms. Circ Res 98: 1123-1133.

6. Pezzolesi MG, Poznik GD, Skupien J, Smiles AM, Mychaleckyj JC, et al (2011) An intergenic region on chromosome $13 q 33.3$ is associated with the susceptibility to kidney disease in type 1 and 2 diabetes. Kidney Int 80: 105111

7. Huang W, Gallois Y, Bouby N, Bruneval P, Heudes D, et al. (2001) Genetically increased angiotensin l-converting enzyme level and renal complications in the diabetic mouse. Proc Natl Acad Sci USA 98: 13330-13334.

8. Takahashi N, Hagaman JR, Kim HS, Smithies O (2003) Minireview: computer simulations of blood pressure regulation by the renin-angiotensin system. Endocrinology 144: 2184-2190.

9. Kakoki M, Takahashi N, Jennette JC, Smithies O (2004) Diabetic nephropathy is markedly enhanced in mice lacking the bradykinin B2 receptor. Proc Natl Acad Sci USA 101: 13302-13305.

10. Breyer MD, Bottinger E, Brosius FC 3rd, Coffman TM, Harris RC, et al. (2005) Mouse models of diabetic nephropathy. J Am Soc Nephrol 16: 27-45.
11. Brosius FC 3rd, Alpers CE, Bottinger EP, Breyer MD, Coffman TM, et al. (2009) Mouse models of diabetic nephropathy. J Am Soc Nephrol 20: 2503-2512.

12. Kakoki M, Sullivan KA, Backus C, Hayes JM, Oh SS, et al. (2010) Lack of both bradykinin B1 and B2 receptors enhances nephropathy, neuropathy, and bone mineral loss in Akita diabetic mice. Proc Natl Acad Sci USA 107: 10190-10195.

13. Kakoki M, Smithies O (2009) The kallikrein-kinin system in health and in diseases of the kidney. Kidney Int 75: 1019-1030.

14. Li F, Wang CH, Wang JG, Thai T, Boysen G, et al. (2010) Elevated tissue factor expression contributes to exacerbated diabetic nephropathy in mice lacking eNOS fed a high fat diet. J Thromb Haemost 8: 2122-2132.

15. Nakagawa T, Sato W, Glushakova O, Heinig M, Clarke T, et al. (2007) Diabetic endothelial nitric oxide synthase knockout mice develop advanced diabetic nephropathy. J Am Soc Nephrol 18: 539-550.

16. Zhao HJ, Wang S, Cheng H, Zhang MZ, Takahashi T, et al. (2006) Endothelial nitric oxide synthase deficiency produces accelerated nephropathy in diabetic mice. J Am Soc Nephrol 17: 2664-2669.

17. Miyamoto Y, Saito Y, Kajiyama N, Yoshimura M, Shimasaki Y, et al. (1998) Endothelial nitric oxide synthase gene is positively associated with essential hypertension. Hypertension 32: 3-8.

18. Onkelinx S, Cornelissen V, Defoor J, Matthijs G, Thomaes T, et al. (2011) The CAREGENE study: genetic variants of the endothelium and aerobic power in patients with coronary artery disease. Acta Cardiol 66: 407-414.

19. Li YY, Zhai ZG, Yang YH, Pang BS, Wang HY, et al. (2011) Association of the $894 \mathrm{G}>\mathrm{T}$ polymorphism in the endothelial nitric oxide synthase gene with risk of venous thromboembolism in Chinese population. Thromb Res 127: 324-327.

20. Noiri E, Satoh H, Taguchi J, Brodsky SV, Nakao A, et al. (2002) Association of eNOS Glu298Asp polymorphism with end-stage renal disease. Hypertension 40: $535-540$.

21. Zeng Z, Li L, Zhang Z, Li Y, Wei Z, et al. (2010) A meta-analysis of three polymorphisms in the endothelial nitric oxide synthase gene (NOS3) and their effect on the risk of diabetic nephropathy. Hum Genet 127: 373-381.

22. Tsukada T, Yokoyama K, Arai T, Takemoto F, Hara S, et al. (1998) Evidence of association of the ecNOS gene polymorphism with plasma NO metabolite levels in humans. Biochem Biophys Res Commun 245: 190-193.

23. He Y, Fan Z, Zhang J, Zhang Q, Zheng M, et al. (2011) Polymorphisms of eNOS gene are associated with diabetic nephropathy: a meta-analysis. Mutagenesis 26: 339-349.

24. Miyamoto Y, Saito Y, Nakayama M, Shimasaki Y, Yoshimura T, et al. (2000) Replication protein $\mathrm{A} 1$ reduces transcription of the endothelial nitric oxide synthase gene containing a -786T-->C mutation associated with coronary spastic angina. Hum Mol Genet 9: 2629-2637.

25. Tervaert TW, Mooyaart AL, Amann K, Cohen AH, Cook HT, et al. (2010). J Am Soc Nephrol 21: 556-563.

26. Adler S, Nast C, Artishevsky A (1993) Diabetic nephropathy: pathogenesis and treatment. Annu Rev Med 44: 303-315.

27. Alpers CE, Hudkins KL (2011) Mouse models of diabetic nephropathy. Curr Opin Nephrol Hypertens 20: 278-284.

28. Walker JD, Close CF, Jones SL, Rafftery M, Keen H, et al. (1992) Glomerular structure in type-1 (insulin-dependent) diabetic patients with normo- and microalbuminuria. Kidney Int 41: 741-748.

29. Selby JV, FitzSimmons SC, Newman JM, Katz PP, Sepe S, et al. (1990) The natural history and epidemiology of diabetic nephropathy. Implications for prevention and control. Jama 263: 1954-1960.

30. Kim Y, Kleppel MM, Butkowski R, Mauer SM, Wieslander J, et al. (1991) Differential expression of basement membrane collagen chains in diabetic nephropathy. Am J Pathol 138: 413-420.

31. Mauer SM, Steffes MW, Ellis EN, Sutherland DE, Brown DM, et al. (1984) Structural-functional relationships in diabetic nephropathy. J Clin Invest 74 1143-1155.

32. Steffes MW, Osterby R, Chavers B, Mauer SM (1989) Mesangial expansion as 
a central mechanism for loss of kidney function in diabetic patients. Diabetes 38: 1077-1081.

33. Steffes MW, Bilous RW, Sutherland DE, Mauer SM (1992) Cell and matrix components of the glomerular mesangium in type I diabetes. Diabetes 41: 679684

34. Najafian B, Mauer M (2009) Progression of diabetic nephropathy in type 1 diabetic patients. Diabetes Res Clin Pract 83: 1-8.

35. Najafian B, Kim Y, Crosson JT, Mauer M (2003) Atubular glomeruli and glomerulotubular junction abnormalities in diabetic nephropathy. J Am Soc Nephrol 14: 908-917.

36. Bohle A, Wehrmann M, Bogenschutz O, Batz C, Muller CA, et al. (1991) The pathogenesis of chronic renal failure in diabetic nephropathy. Investigation of 488 cases of diabetic glomerulosclerosis. Pathol Res Pract 187: 251-259.

37. Harris RD, Steffes MW, Bilous RW, Sutherland DE, Mauer SM, et al. (1991) Global glomerular sclerosis and glomerular arteriolar hyalinosis in insulin dependent diabetes. Kidney Int 40: 107-114

38. Stout LC, Kumar S, Whorton EB (1994) Insudative lesions--their pathogenesis and association with glomerular obsolescence in diabetes: a dynamic hypothesis based on single views of advancing human diabetic nephropathy. Hum Pathol 25: 1213-1227.

39. Alsaad KO, Herzenberg AM (2007) Distinguishing diabetic nephropathy from other causes of glomerulosclerosis: an update. J Clin Pathol 60: 18-26.

40. Kanetsuna Y, Takahashi K, Nagata M, Gannon MA, Breyer MD, et al. (2007) Deficiency of endothelial nitric-oxide synthase confers susceptibility to diabetic nephropathy in nephropathy-resistant inbred mice. Am J Pathol 170: 14731484.

41. Albrecht EW, Stegeman CA, Heeringa $\mathrm{P}$, Henning $\mathrm{RH}$, van Goor $\mathrm{H}$, et al. (2003) Protective role of endothelial nitric oxide synthase. J Pathol 199: 8-17.

42. Kubes $P$, Suzuki M, Granger DN (1991) Nitric oxide: an endogenous modulator of leukocyte adhesion. Proc Natl Acad Sci U S A 88: 465-4655.

43. Freedman JE, Sauter R, Battinelli EM, Ault K, Knowles C, et al. (1999) Deficient platelet-derived nitric oxide and enhanced hemostasis in mice lacking the NOSIII gene. Circ Res 84: 1416-1421.

44. Jojima T, Suzuki K, Hirama N, Uchida K, Hattori Y, et al. (2009) Glimepiride upregulates eNOS activity and inhibits cytokine-induced NF-kappaB activation through a phosphoinoside 3-kinase-Akt-dependent pathway. Diabetes Obes Metab 11: 143-149.
45. Aoki R, Ikarugi H, Naemura A, ljiri Y, Yamashita T, et al. (2006) Endothelia dysfunction precedes atherosclerotic lesions and platelet activation in high fat diet-induced prothrombotic state. Thromb Res 117: 529-535.

46. Sakai T, Nambu T, Katoh M, Uehara S, Fukuroda T, et al. (2009) Up-regulation of protease-activated receptor-1 in diabetic glomerulosclerosis. Biochem Biophys Res Commun 384: 173-179.

47. Wang CH, Li F, Hiller S, Kim HS, Maeda N, et al. (2011) A modest decrease in endothelial NOS in mice comparable to that associated with human NOS3 variants exacerbates diabetic nephropathy. Proc Natl Acad Sci U S A 108: 2070-2075.

48. Nakagawa T (2008) Uncoupling of VEGF with $N O$ as a mechanism for diabetic nephropathy. Diabetes Res Clin Pract 1: 67-69.

49. Nakagawa T, Johnson RJ (2011) Endothelial nitric oxide synthase. Contrib Nephrol 170: 93-101.

50. Nakagawa T (2009) A new mouse model resembling human diabetic nephropathy: uncoupling of VEGF with eNOS as a novel pathogenic mechanism. Clin Nephrol 71: 103-109.

51. Sato W, Kosugi T, Zhang L, Roncal CA, Heinig M, et al. (2008) The pivotal role of VEGF on glomerular macrophage infiltration in advanced diabetic nephropathy. Lab Invest 88: 949-961.

52. Kosugi T, Heinig M, Nakayama T, Connor T, Yuzawa Y, et al. (2009) Lowering blood pressure blocks mesangiolysis and mesangial nodules, but not tubulointerstitial injury, in diabetic eNOS knockout mice. Am J Pathol 174 1221-1229.

53. Kosugi T, Heinig M, Nakayama T, Matsuo S, Nakagawa T, et al. (2010) eNOS knockout mice with advanced diabetic nephropathy have less benefit from renin-angiotensin blockade than from aldosterone receptor antagonists. Am J Pathol 176: 619-629.

54. Zhang MZ, Wang S, Yang S, Yang H, Fan X, et al. (2012) Role of blood pressure and the renin-angiotensin system in development of diabetic nephropathy (DN) in eNOS -/- db/db mice. Am J Physiol Renal Physiol 302: 433-438.

55. Kuno Y, lyoda M, Shibata T, Hirai Y, Akizawa T, et al. (2011) Sildenafil, a phosphodiesterase type 5 inhibitor, attenuates diabetic nephropathy in noninsulin-dependent Otsuka Long-Evans Tokushima Fatty rats. Br J Pharmaco 162: $1389-1400$ 